\title{
EMPIRICAL DETERMINATION OF OPTIMAL PARAMETERS FOR SODIUM DOUBLE-EDGE MAGNETO-OPTIC FILTERS
}

\author{
Ian F. Barry ${ }^{1 *}$, Wentao Huang ${ }^{1}$, John A. Smith ${ }^{1}$, and Xinzhao Chu ${ }^{1}$ \\ ${ }^{I}$ Cooperative Institute for Research in Environmental Sciences \& Department of Aerospace Engineering \\ Sciences, University of Colorado Boulder, CO80309,USA, EEmail:Ian.Barry@colorado.edu
}

\begin{abstract}
A method is proposed for determining the optimal temperature and magnetic field strength used to condition a sodium vapor cell for use in a sodium Double-Edge Magneto-Optic Filter (Na-DEMOF). The desirable characteristics of these filters are first defined and then analyzed over a range of temperatures and magnetic field strengths, using an IDL Faraday filter simulation adapted for the Na-DEMOF. This simulation is then compared to real behavior of a Na-DEMOF constructed for use with the Chu Research Group's STAR Na Doppler resonance-fluorescence lidar for lower atmospheric observations.
\end{abstract}

\section{INTRODUCTION}

The Na-DEMOF has previously demonstrated a capacity for high-resolution temperature and wind measurements in the stratosphere and lower mesosphere [3]. However, processing these measurements requires calibration that assumes vertical winds that average to zero, resulting in a set of calibration constants that vary on a nightly or even an hourly basis. Figure 1 shows the result of deriving temperature and vertical wind after calibrating the system every 30 minutes and integrating over the 4-hour collection period. Using the average calibration over the entire collection period results in a negative $3-10 \mathrm{~K}$ bias in temperature. Reliably calibrated data can only be obtained by reducing this instability, which in turn necessitates the identification and stabilization of the factors involved. To this end, the theoretical and practical variables that are known to affect Na-DEMOF performance will be described in terms of their stability and effects on the filter function.

The four theoretical factors that have been identified as influential on vapor cell filter performance are: vapor density, vapor temperature, optical thickness, and magnetic field strength. The simulation developed by S. D. Harrell and modified by Wentao Huang to calculate the absorption and dispersion of orthogonal circular polarizations of light within a $\mathrm{Na}$ vapor cell allows for calculation of the DEMOF filter function by specifying each of these variables $[1,2]$. Vapor density is determined by controlling the temperature at which a reservoir of solid $\mathrm{Na}$ is held, and optical thickness is determined by this vapor density and the physical length of the cell.
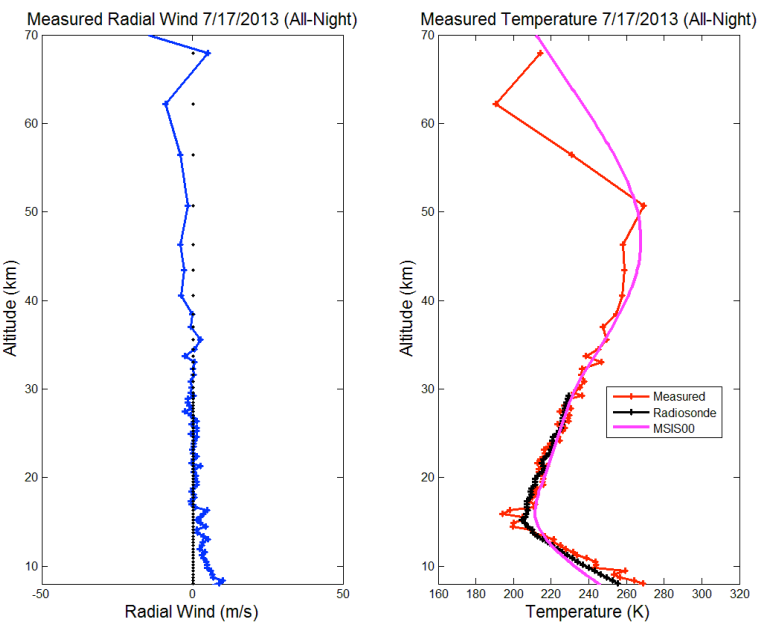

Fig. 1. Measurements of temperature and vertical wind achieved from 10 to $65 \mathrm{~km}$ using 30-minute calibration scheme.

The additional practical considerations that have been found to affect Na-DEMOF performance since its construction are: vapor cell window depolarization caused by stress creating scattered areas of disorganized birefringence, $\mathrm{Na}$ condensation on the vapor cell windows, and oxidation of the solid $\mathrm{Na}$ reservoir due to imperfections in vapor cell vacuum. The window depolarization results in a constant efficiency reduction but can be avoided by loosening the structure holding the windows, and the window condensation has been mitigated by locating the 
heaters near the windows and occasionally evaporating the condensate from the outside using a heat gun. Oxidation causes a progressive reduction of $\mathrm{Na}$ vapor availability requiring higher temperatures to produce the desired amount of vapor, and new manufacturing methods are being employed to improve the vacuum and resultant vapor cell lifetimes.

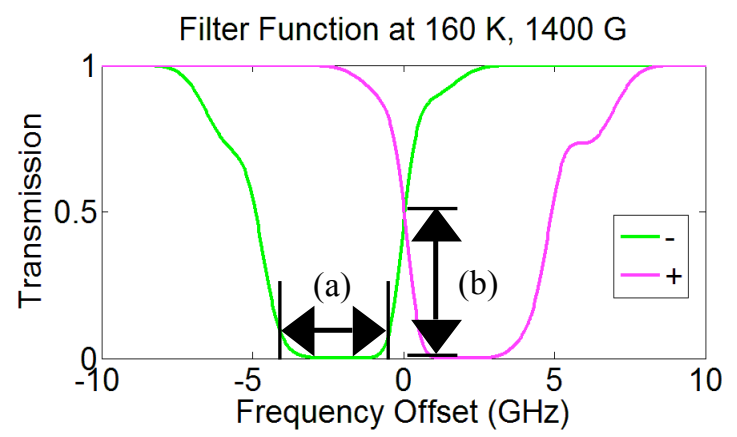

Fig. 2. Example filter function with temperature at $160 \mathrm{~K}$ and magnetic field strength at $1400 \mathrm{G}$. (a) shows the trough width and (b) shows the crossover height. The edge slope is determined by the slopes in the region within $0.5 \mathrm{GHz}$ of the crossover point.

Figure 2 provides an example of an ideal simulated Na-DEMOF filter function for lower altitude wind and temperature derivations. In order to provide the optimal sensitivities for useful data acquisition, the transmitted laser frequencies must lie within the region shown in the center of the figure, where the two channels of the DEMOF act as sloped edge filters. This is best achieved by tuning the temperature and magnetic field used to condition the vapor cell such that the crossover point occurs near $50 \%$ transmission, the edge of the filter are sloped gradually enough to accommodate the transmitted laser wavelengths, and the transmission runs from near $100 \%$ outside the filter region to near zero within the trough. To determine the performance of a Na-DEMOF filter, these characteristics have been defined as crossover height, edge slope, and trough width.

Ideally, a Na-DEMOF should also maintain stable operation for extended periods of time, requiring calibration no more frequently than once per night and replacement after no less than a month for reasonable operability. The final paper will demonstrate these stabilities and the derived atmospheric winds and temperatures, including analysis of the sensitivity of the derived results to slight errors in calibration.

Later on we discovered that the hourly or halfhourly variations of calibration factors were caused by the operators' periodic rotation of a quarter wave plate used in the Na-DEMOF receiver setup. Such manual rotation of the quarter wave plate could not ensure a stable calibration of polarization states, resulting in varied calibration factors depending on the orientation angle of the quarter wave plate. Once the quarter wave plate was fixed to an angle (supposedly the optimum orientation), the calibration factor became constant throughout the night observations.

\section{METHODOLOGY}

Design considerations limit the difference that may be achieved between the vapor temperature and the "tip temperature" for the solid $\mathrm{Na}$ reservoir controlling the vapor density, and in the cell designed by Wentao Huang the same temperature controller is used for both. This simplifies the theoretical problem to one dependent only on temperature, magnetic field strength, and vapor cell length. The temperatures of the Na reservoir and vapor within the cell are not determined directly, but due to the insulation around the vapor cell can be assumed to lie steadily within 2-5 K of the control temperature, with heaters located near the windows and temperature measured by an RTD near the center of the cell. The control temperature can be set and obtained within $0.1 \mathrm{~K}$, and length is fixed but can be determined accurately to within a fraction of a millimeter. However, the magnetic field is neither constant, as assumed by the simulation, nor easily characterized. Similarly, the vapor density is affected by the age of the vapor cell, the mass of solid $\mathrm{Na}$ present in the reservoir, and the insulation-dependent temperature distribution around the cell. Therefore, the filter function must be tested over a range of temperatures in order to empirically determine the operating temperature which produces a crossover height of $50 \%$ transmission.

In order to guide this testing, it is important to have an idea of how temperature and magnetic field strength affect the filter function. To this end, the Na-DEMOF simulation code was used to determine the trough widths, edge slopes, and 
crossover heights of filter functions for ideal vapor cells held at various temperatures within various strengths of magnetic field.

\section{RESULTS}

Figures 3-5 show the dependence of the three key filter function characteristics on temperature and magnetic field strength. Trough width was found to increase with increasing temperature, edge slope was found to decrease with increasing temperature except at a magnetic field strength range between 1350-1400 Gauss, and crossover height was found to decrease with increasing temperature. Figure 5 can also be used to determine the effective magnetic field strength of a Na-DEMOF filter once the optimal temperature producing $50 \%$ transmission crossover height has been achieved.

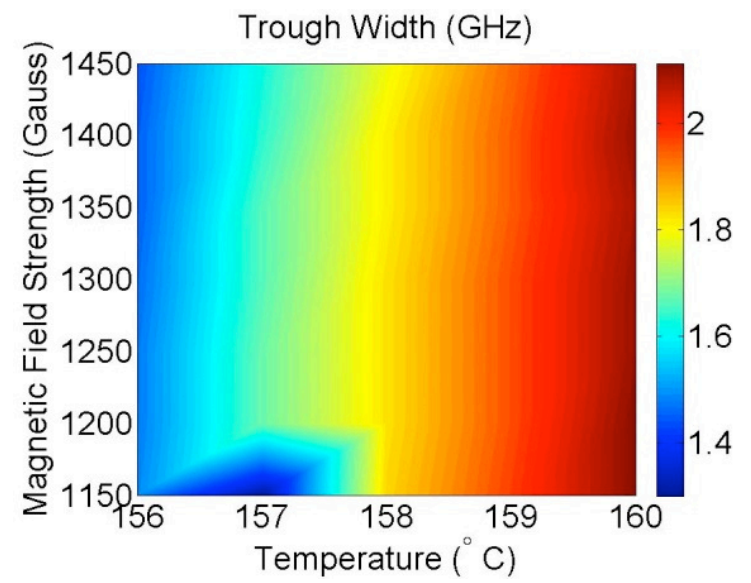

Fig. 3. Dependence of trough width on temperature and magnetic field strength.

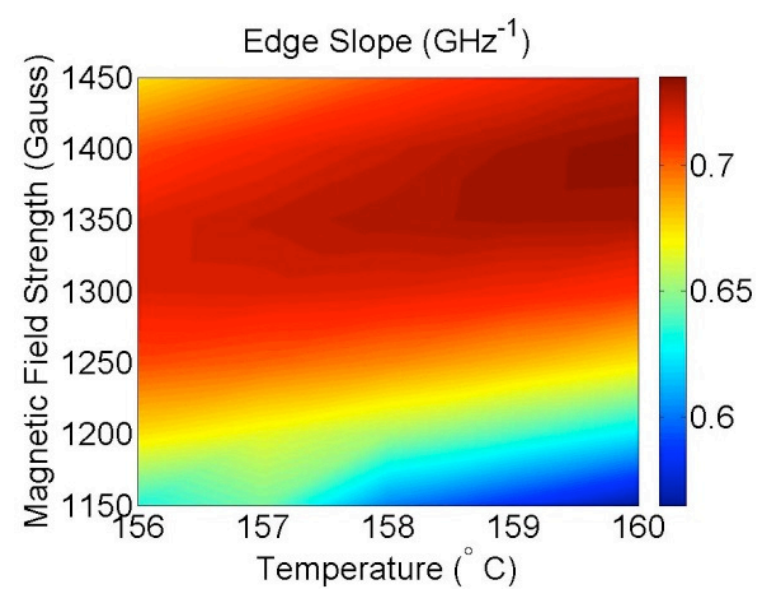

Fig. 4. Dependence of edge slope on temperature and magnetic field strength.

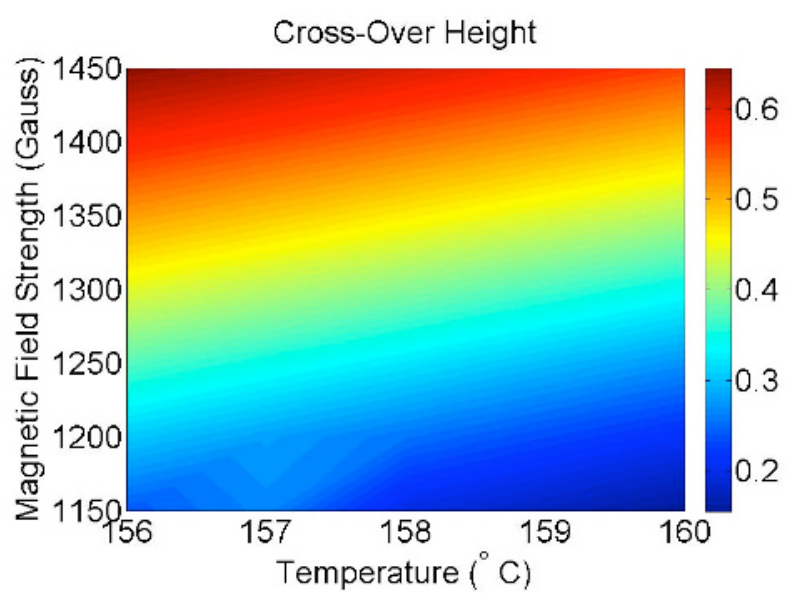

Fig. 5. Dependence of crossover height on temperature and magnetic field strength.

\section{CONCLUSIONS}

For the greatest sensitivity determined by edge slope, a temperature of $160^{\circ} \mathrm{C}$ or hotter and a magnetic field strength of around 1400 Gauss is ideal. However, for a broader edge filter function allowing the widest range of transmitted laser wavelengths, lower magnetic field strengths or higher temperatures may be required. In either case, higher temperatures provide more complete rejection within the function trough and thus more effective return signal frequency dependence. However, sensitivity from high temperatures must be traded off with cell lifetime degradation from oxidation and condensation, so the lowest possible temperature for a given application will grant the longest lifetime. The results of a filter optimized using this method will be presented in the final paper, demonstrating derivation of temperature and zonal and meridional winds from the stratosphere throughout the mesosphere and lower thermosphere.

\section{ACKNOWLEDGEMENT}

We acknowledge the support of a NSF CRRL grant (AGS-1136272) and CEDAR grant (AGS1042257). I.B. was partially supported by PLR1246405. 


\section{REFERENCES}

[1] Harrell, S. D., C.-Y. She, T. Yuan, D. A. Krueger, H. Chen, S. S. Chen, Z. L. Hu, 2009: Sodium and potassium vapor Faraday filters revisited: theory and applications, J. Opt. Soc. Am., 26(4), 659-670.

[2] Huang, W., X. Chu, B. P. Williams, S. D. Harrell, J. Wiig, C. Y. She, 2009: Na double-edge magneto-optic filter for $\mathrm{Na}$ lidar profiling of wind and temperature in the lower atmosphere, Optics Letters, 34(2), 199-201.

[3] Huang, W., X. Chu, J. Wiig, B. Tan, C. Yamashita, T. Yuan, J. Yue, S. D. Harrell, C.-Y. She, B. P. Williams, J. S. Friedman, R. M. Hardesty, 2009: Field demonstration of simultaneous wind and temperature measurements from 5 to $50 \mathrm{~km}$ with a Na double-edge magnetooptic filter in a multi-frequency Doppler lidar, Optics Letters, 34(10), 1552-1554.

[4] Schreier, F., 1992: The Voigt and Complex Error Function: A Comparison of Computational Methods, J. Quant. Spectrosc. Radiat. Transfer, 48(5/6), 743-763. O'Connor, E. J., A. J. Illingworth, R. J. Hogan, 2004: A technique for autocalibration of cloud lidar, J. Atmos. Ocean Tech., 21(5), 777-778. 